\title{
Numerical investigations of shape of the reflecting surface made of knitted mesh fabric being pulled on the curvilinear frame
}

\author{
Vitaly Meshkovsky ${ }^{1, *}$, Anatoly Sdobnikov ${ }^{1}$, Sergey Churilin ${ }^{1}$, and Yuri Kisanov ${ }^{2}$ \\ ${ }^{1}$ Bauman Moscow State Technical University, \\ 105005 2-nd Baumanskaya str. 5 building 1, Moscow, Russia \\ ${ }^{2}$ JSC Academician M.F. Reshetnev Information Satellite Systems, Moscow office, \\ 662972 Lenin Street 52, Zheleznogorsk, Krasnoyarsk region, Russia
}

\begin{abstract}
This paper presents the surface shaping numerical investigations results of truss space constructions mesh reflectors, such as antennas and calibration and adjustment satellites. Shape-generating structure of mentioned constructions adds up to set of triangular facets, made in the form of spatio-curvilinear bar frames, bearing reflecting knitted mesh fabric pulled on it. This work proposes the algorithm of calculation of step-by-step reflecting mesh pulling on the bearing frame's bars process, using finite elements method. Numerical execution of the developed algorithm involves for resolving the linear elasticity theory firsttype boundary value problem, which implies integration of elastic body equilibrium equations without taking into account mass forces when kinematic boundary conditions are given. Analyzing when having done numerical calculations, it's possible to determine what grade obtained shapes of reflecting surfaces are precise with, and to find possible for developing variants of the antenna structure, which would allow to obtain the reflector surface shape with required accuracy by using flexible cables as a part of shape-generating structure. Comparing results of numerical investigations with experimental data received using full-scaled model of spherical calibration and adjustment satellite shows satisfactory qualitative and quantitative matching of both results.
\end{abstract}

\section{Introduction}

Increasing necessity of transformable reflectors with aperture of more than $12 \mathrm{~m}$, which are used for telecommunication applications, compels such structures developers to find the best conceptual decisions that would meet the demands of minimal mass, high precision of reflecting surface, and necessary dynamic stiffness [1-3].

Modern technology of creating large-scaled structures is based on the module paradigm. This technology allows building in space rather large, one might say giant multikilometer

\footnotetext{
*Corresponding author: vitevm@yandex.ru
} 
frameworks of space vehicles, antenna reflectors, basic structures of solar power stations, large telescopes etc. It's possible to build all such constructions on the basis of frame modules.

During a near of semi-centennial history of the large space self-deployable reflectors technology evolution, dozens of variants of their structures have been put forward, but to the purposes of practical usage it makes sense to consider two of structure types, which vary in methods of reflecting surface shaping, that are

- frame structures, where flexible reflecting surface is fixed to the load-bearing frame, forming required shape;

- shell-based structures, where reflecting surface is made as a result of elastic deformations arising either by the assembly forces, or by the membrane forces when there is excess pressure in the closed shell.

If this is the case that mechanical or else frame structures present almost the whole set of reflectors currently running in space, there are 50 of 60 , or 83 percent with the sizes within the range of 4.5 to 22 meters, then part of elastic reflectors contains only 10 ones, at that, all of the elastic reflectors have dimensions of less than 7 meters. Inflatable constructions holding their much promise stay under development for now.

Currently, in world practice there are four companies commanding in development of transformable reflectors, which develop frame reflectors with mesh reflecting surfaces. As of abroad, there are two U.S. companies, namely Harris and NGST, which one is subdivision of Northrop Grumman corp., and Toshiba the Japanese company one. In Russia, JSC Academician M.F. Reshetnev Information Satellite Systems company takes leading position in development of transformable reflectors.

Harris develops umbrella-type scheme of reflector frame building (Radial Rib, Folding Rib) (Fig. 1a) [4, 5] and hoop-similar structure as of a last variant (Fig. 1b) (Harris Advanced Hoop-Truss Reflector).

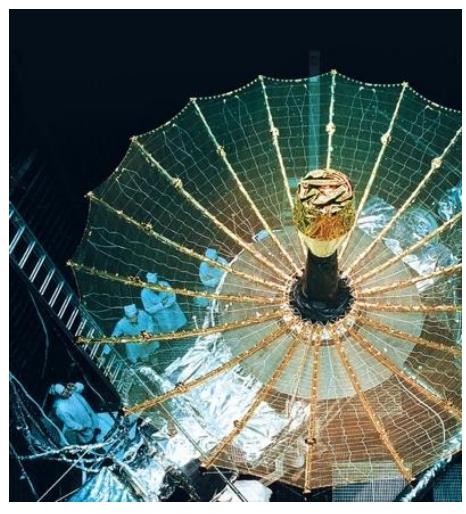

a)

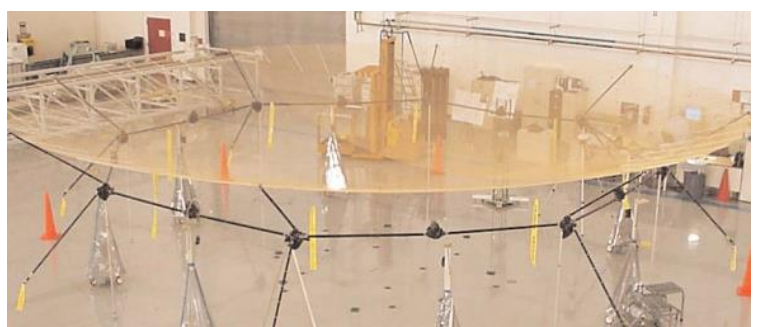

b)

Fig. 1. Umbrella-type and hoop-type schemes of reflectors by Harris.

The hoop-type structure was developed as alternative to the umbrella-type one. Initial investigations of the structure were provided by Northrop Grumman Corp. Hoop-type reflector consists of peripheral hoop, two meshes, and inner cable web interconnecting the meshes. One of the meshes is a shaping mesh, and reflecting surface is fixed to it (Fig. 2a) [6]. Further investigation have led to developing of large deployable hoop-type reflector with diameter of 11.5 meters as a specimen of "double pantograph" concept for BIOMASS mission (Fig. 2b) [7]. 
JSC Academician M.F. Reshetnev Information Satellite Systems company works on umbrella-type reflector concept (Fig. 3) [8, 9]. Currently, reflectors of cable-stayed umbrella-type structure with larger diameters are under development.

Frame reflectors engineering are carried out by another more companies. RSC Energia under the treaty with ESA tries to implement a concept of reflector of cablestayed umbrella-type structure with the circular peripheral pantograph and meshed reflecting surface [4].

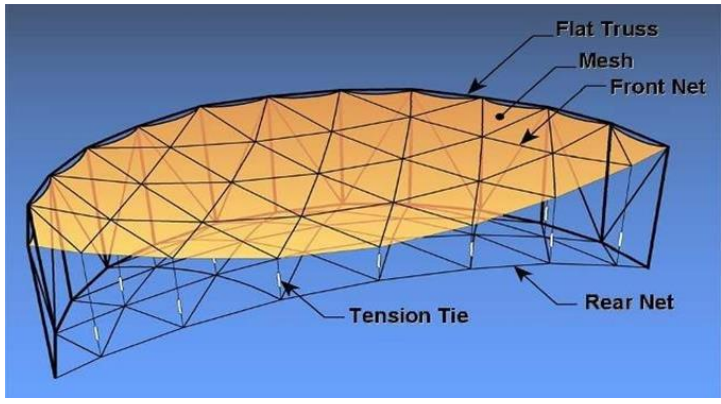

a)

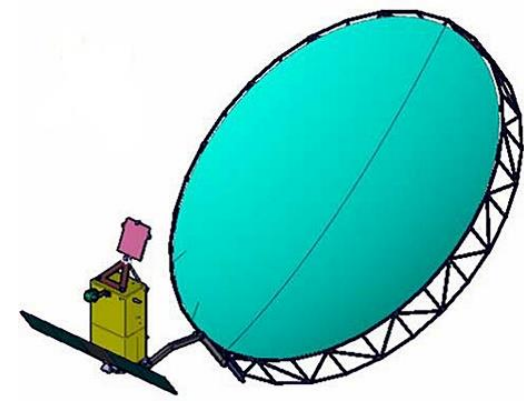

b)

Fig. 2. Structure of hoop-type reflectors by Northrop Grumman Corporation (AstroMesh structure).

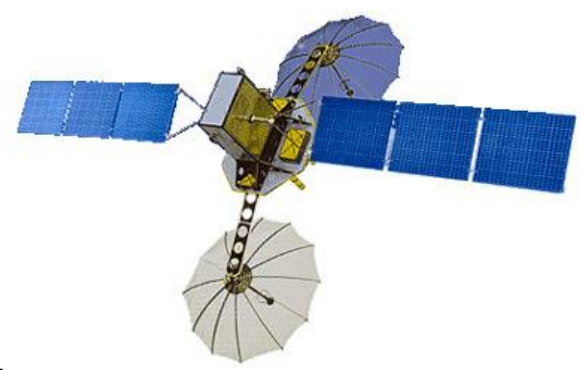

Fig. 3. Reflectors with diameter of $4.5 \mathrm{~m}$ at Luch $5 \mathrm{~A}$ satellite.

Truss reflectors development has been started simultaneously with umbrella-type ones on $60^{\text {th }}$ of last century. In USA, General Dynamics company on demand of NASA carried out the development, results of which were published in the shortening way in the article [10]. Next years, various conceptual nature works on the possibility of large reflectors creating have started publishing, the truss structures were under consideration among them.

Special Design Bureau of the Moscow Power Engineering Institute for years has been developing a trend of truss framework with basic element in the form of tetrahedron and with the meshed reflecting surface [12]. Truss reflector structures like that have been developed and set among other at Kondor satellite by NPO Mashinostroyeniya [13] (Fig. 4a) and at China satellite of HJ-1C (Fig. 4b).

Japan Aerospace Exploration Agency (JAXA) advances mesh modules based paradigm. (Fig. 5) [4], [14-18].

This paper presents truss structures, which are, in particular, the base elements of antenna reflectors and calibration and adjustment satellites with the mechanical foldable frames and reflecting surfaces made of knitted mesh fabric fixed to it. When considering such structures, special interest makes an accuracy of reflecting surface approximation relative to theoretical shape. 
The purpose of this work is to perform the numerical investigations of shape of the reflecting surface made of knitted mesh fabric being pulled on the truss structure curvilinear frame.

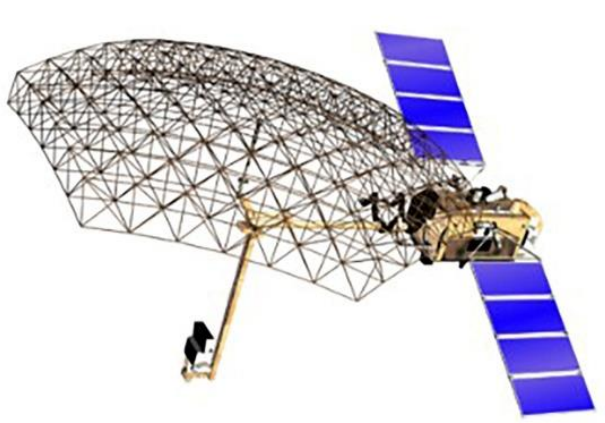

a)

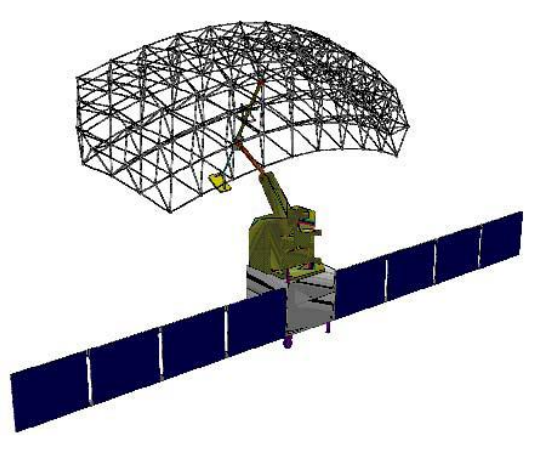

b)

Fig. 4. Space satellites with truss reflectors.

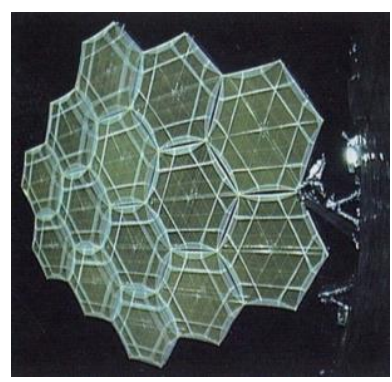

a)

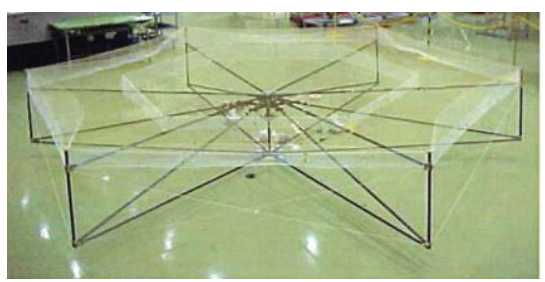

b)

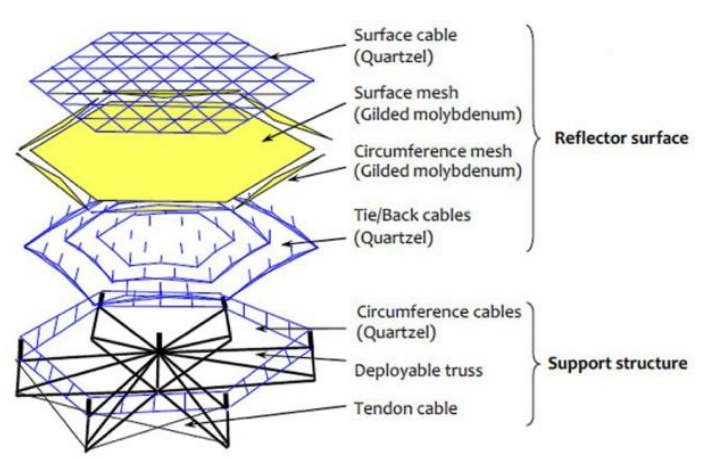

c)

Fig. 5. Truss structure named as "modular mesh":

a) large deployable reflector with diameter of $19 \mathrm{~m}$ consists of 14 hexahedral unfurlable modules,

b) mesh module with diameter of $4.8 \mathrm{~m}$,

c) structure of the mesh module.

\section{Construction of the truss module of a space system}

The key element of reflector frame is the repetitive volumetric element that is tetrahedron (Fig. 6a). At that, tetrahedrons interconnections are made in such a way that their vertices are placed at facing and rear surfaces, changing their own top and bottom directions by turns. As a result, the volumetric truss structure forms, being built as two surfaces 
interconnected by diagonal rods (Fig. 6b) Structurally, each surface is a package of foldable rods consisting of two joined tube elements. Diagonal and foldable rods are interconnected by nodal hinges, which makes kinematic constraints, thus this allows to consider truss framework as the whole of spatial cells in the form of tetrahedron.

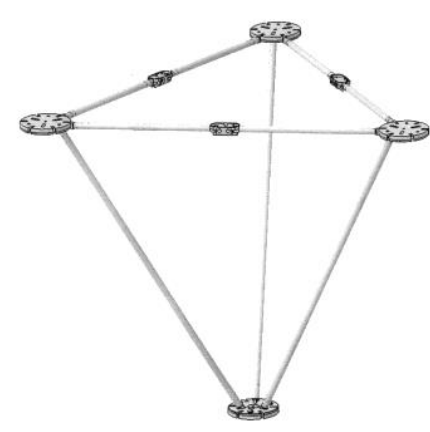

a)

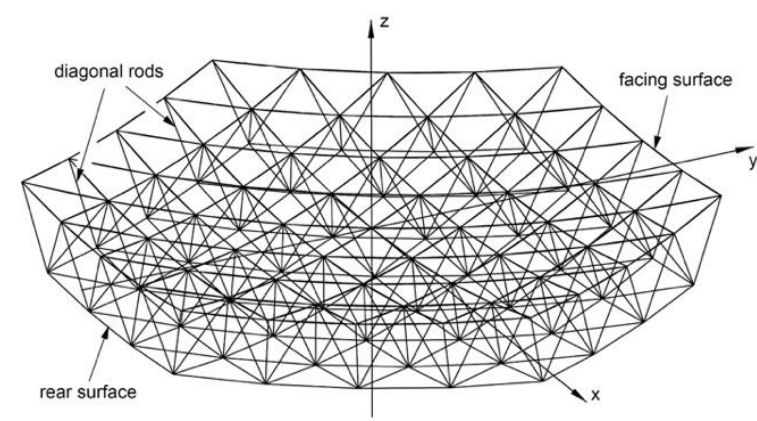

b)

Fig. 6. Truss reflector framework.

The mesh fabric is fixed to the facing surface, it is knitted material made from various metals microwires, such as molybdenum, tungsten, stainless steel ones, which are suitable to be processed on knitted machines and provide required mechanical and radiotechnical characteristics. It should be noted, that tetrahedron faces split ideal facing surface to triangular areas, or else to facets.

Hereinafter, truss module of space antenna with reflector with diameter of $12 \mathrm{~m}$ and with focal length of $12 \mathrm{~m}$ containing $N_{0}=4$ stages is shown as example. In this structure, main foldable bars length is $1.5 \mathrm{~m}$, diagonal bars length is $1.6 \mathrm{~m}$. Antenna aperture is 113.4 $\mathrm{m}^{2}$ and height of arcs is $h=11.7 \mathrm{~mm}$.

When folding, tube elements of foldable rods turn around axes located in the nodal hinges and stand inside the framework between diagonal rods. Last ones turn in the same way about their axes located in the nodal hinges and stand the near parallel position in folded state (Fig. 7).

In orbit, the whole construction deploys by means of elastic energy initially accumulated in springs placed in the nodal hinges and the hinges of foldable rods when construction is being folded. Diameter of the truss module in a close-packed (transport) state is a value by order of 0.7 $\mathrm{m}$. The areas ratio is $0.7 / 113.4=0.0062$.

Generally, the shape-generating structure (SGS) of the truss reflector consists of rigid elements of the framework facing surface (nodes and rods) and flexible structure, which is inside the triangular facets, approximating the theoretical surface.

The possibility of reflecting surface of the reflector to be represented as number of facets by means of rigid elements, probably, is the main advantage of the truss reflectors.

When building SGS using rigid elements, four main advantages put into effect:

- positions of the nodal points of the framework facing surface are set by a building berth, and there is no need for next adjustment,

- SGS is made by facets in the form of triangle, and that is optimal facet form among all geometrical forms,

- using the rigid connecting elements of the facing surface (rods), it is possible to use profiled ones, which increase precision of reflecting surface approximation in two times as compared with using straight rods [19, 20],

- when profiled the facets borders, residual inaccuracies of approximation are independent, they are localized in the centers of triangular facets, therefore they can be decreased by using flexible SGS cables located inside the triangles. 


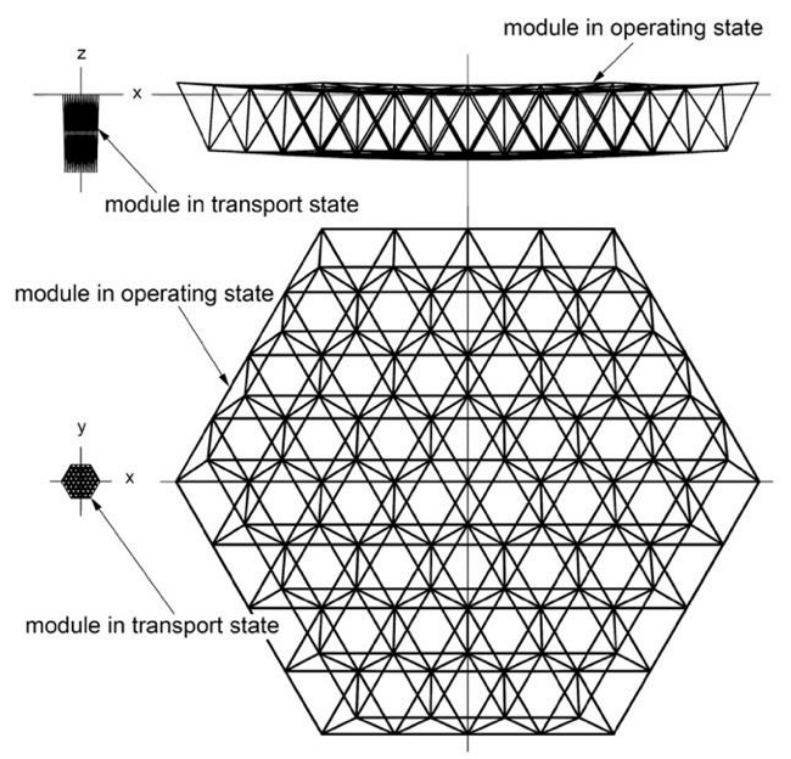

Fig. 7. The truss module in folded transport state and unfolded operating state.

Fig. 8a shows the framework and shape-generating structure elements of a single tetrahedron. In Fig. $8 \mathrm{~b}$ the height of arcs is shown increased in 10 times to better visual comprehension of the curvilinear foldable rods geometry.

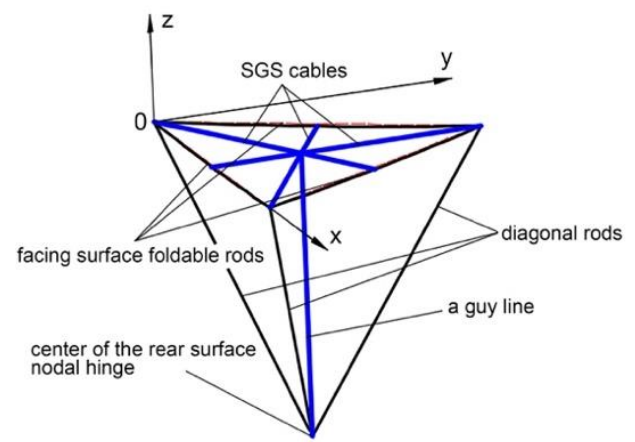

a)

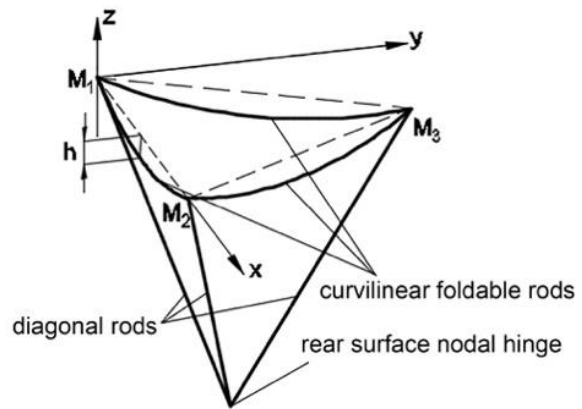

b)

Fig. 8. The framework and the shape-generating structure elements of a single tetrahedron.

In the model being under consideration, it is supposed that middle points of the arcs (that are centers of the foldable rods hinges) and centers of the nodal hinges (that are points $M_{1}, M_{2}$ and $M_{3}$ ) lie on an ideal paraboloid surface, which is theoretical surface of the reflector.

\subsection{Performing of the knitted mesh fabric stress analysis of a reflector framework single facet}

Hereinafter, stress analysis of the knitted mesh fabric being pulled on the curvilinear frame of a single facet is described. Rods of the framework are supposed to be rigid. 
The problem is put as the linear elasticity theory first-type boundary value problem, which implies integration of elastic solid body equilibrium equations without taking into account mass forces (1)

$$
\nabla \cdot \hat{\sigma}=0
$$

where $\hat{\sigma}$ is the Cauchy stress tensor, $\nabla=\frac{\partial}{\partial x_{i}} \boldsymbol{e}_{i}$ is the del operator, $(\cdots \cdots)$ is the dot product,

when kinematic boundary conditions are given (2), that are displacements specified on the body surface $S_{u}$

$$
\boldsymbol{u}=\boldsymbol{u}_{\mathbf{0}}(\boldsymbol{r}) \text {, where } \boldsymbol{r}=x_{\boldsymbol{i}} \boldsymbol{e}_{i} \in S_{u} .
$$

The knitted mesh fabric nesting is in the shape of flat triangle similar to the triangle $M_{1} M_{2} M_{3}$ (Fig. 9). The nesting side that is opposite to the vertex $M_{1}$ lies at distance of $\delta$ from the line $M_{1} M_{2}$, which allows to carry out a computations with various pulling of the mesh.

Three variants, that are without SGS (Fig. 9a), with SGS made of flexible elements meeting in one point (Fig. 9b), and with SGS made of flexible elements coinciding with the facet medians (Fig. 9c), have been considered.

Computations are made with the help of finite element method, using MSC.Patran/Nastran software.

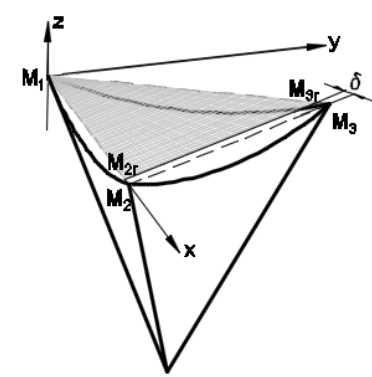

a)

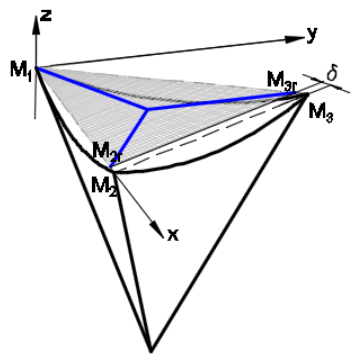

b)

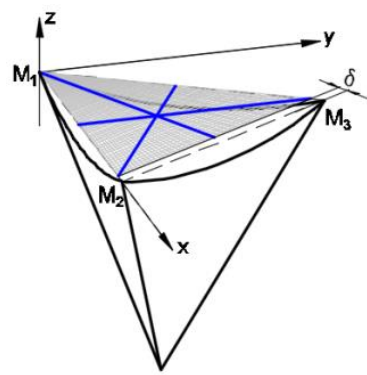

c)

Fig. 9. Variants of SGS implementation.

Finite element model of a single facet (Fig. 10a) is shown in Fig. 10b. The finite element model consists of mesh fabric model divided into Quad4 elements, flexible SGS cables model divided into Bar2 elements, and additional flexible SGS elements, which are guy lines modeled by MPC elements.

Accepted characteristics of the knitted mesh fabric and the SGS cables are shown in Table 1.

Table 1. Physical properties of the knitted mesh fabric and the SGS cables.

\begin{tabular}{|l|c|c|c|}
\hline & $E, \mathrm{~Pa}$ & size, $\mathrm{mm}$ & Grade \\
\hline knitted mesh fabric & $6.0 \cdot 10^{6}$ & 0.15 & Atlas-Atlas 2G \\
\hline SGS cables & $7.18 \cdot 10^{9}$ & 0.70 & Armalon \\
\hline
\end{tabular}

The mesh fabric and the SGS cables pulling is made step-by-step when the finite element model node in the point $M_{1}$ is constrained. Shown below the given problem boundary conditions answer to each step. 


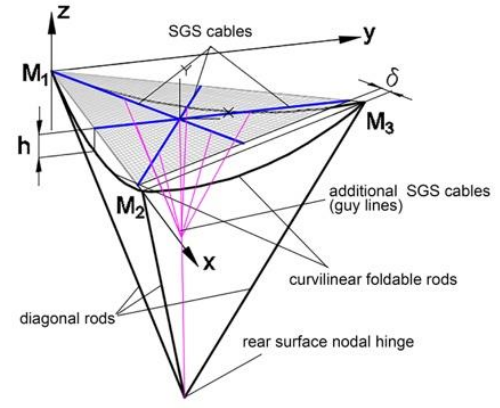

a)

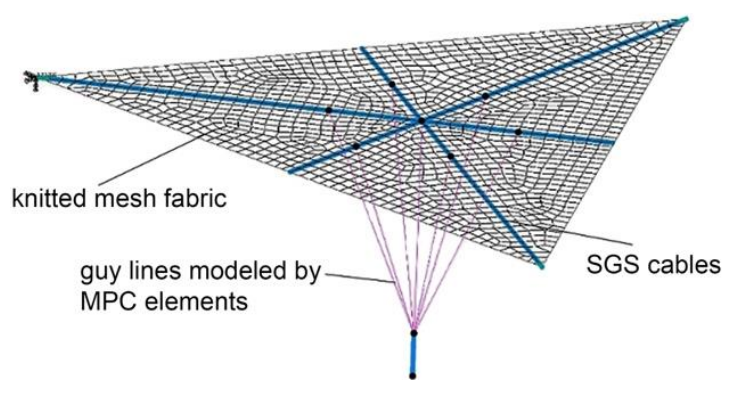

b)

Fig. 10. The single facet of the reflector framework and the finite element model of knitted mesh fabric with SGS cables.

\subsection{Steps of the knitted mesh fabric and the SGS cables pulling}

When performing an analysis, the finite element model node in the point $M_{1}$ is considered to be constrained, which prevent the mesh fabric displacement as a solid body (Fig. 11).

\subsection{1 $1^{\text {st }}$ step}

Displacements of the mesh nodes for the $1^{\text {st }}$ step are shown by arrows in Fig. 11, which corresponds to the mesh fabric being pulled in the plane of triangle $M_{1} M_{2} M_{3}$.

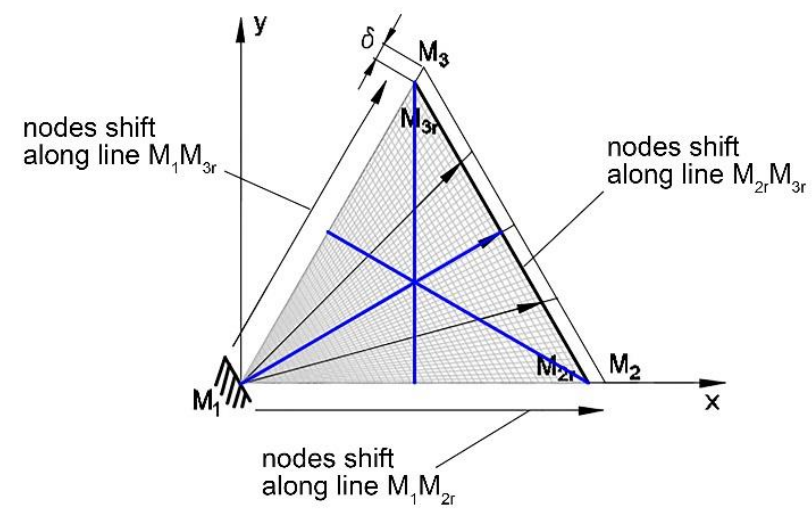

Fig. 11. $1^{\text {st }}$ step of the knitted mesh fabric deformation.

Nodes $M_{i}$ that belong to line $M_{1} M_{2 r}$ shift along line $M_{1} M_{2}$ in compliance with the law of (3)

$$
\left.\begin{array}{c}
u_{1 i}^{(1)}=\frac{x_{1 i}^{(0)}}{L_{1}^{(0)}} \delta \\
v_{1 i}^{(1)}=0 \\
w_{1 i}^{(1)}=0
\end{array}\right\}
$$


where $x_{1 i}^{(0)}$ is the abscissa of the node $M_{i}, L_{1}^{(0)}$ is the length of the line $M_{1} M_{2 r}$.

Accepted law of movement of nodes $M_{i}$ is similar to shifting of elastic rod sections while stretched along their axis.

Nodes $M_{k}$ that belong to line $M_{2 r} M_{3 r}$ shift along rays derived from point $M_{1}$ in compliance with the law of (4)

$$
\left.\begin{array}{c}
u_{2 k}^{(1)}=x_{2 k}^{(1)}-x_{2 k}^{(0)} \\
v_{2 k}^{(1)}=y_{2 k}^{(1)}-y_{2 k}^{(0)} \\
w_{2 k}^{(1)}=0
\end{array}\right\}
$$

where

$$
\begin{gathered}
x_{2 k}^{(1)}=x_{2 k}^{(0)} \frac{L_{2 k}^{(1)}}{L_{2 k}^{(0)},} y_{2 k}^{(1)}=y_{2 k}^{(0)} \frac{L_{2 k}^{(1)}}{L_{2 k}^{(0)}} \\
L_{2 k}^{(0)}=\sqrt{\left(x_{2 k}^{(0)}\right)^{2}+\left(y_{2 k}^{(0)}\right)^{2}}, L_{2 k}^{(1)}=L_{2 k}^{(0)}+L_{2 k}^{(0)}\left(\frac{L_{1}^{(1)}}{L_{1}^{(0)}}-1\right) .
\end{gathered}
$$

Nodes $M_{j}$ that belong to line $M_{1} M_{3 r}$ shift along line $M_{1} M_{3}$ in compliance with the law of (5)

$$
\begin{gathered}
\left.\begin{array}{c}
u_{3 j}^{(1)}= \\
v_{3 j}^{(1)}=\frac{L_{3 j}^{(0)}}{L_{3}^{(0)}}\left(L_{3}^{(1)}-L_{3}^{(0)}\right) \frac{x_{3}}{L_{3}^{(1)}}\left(L_{3}^{(1)}-L_{3}^{(0)}\right) \frac{y_{3}}{L_{3}^{(1)}} \\
w_{3 j}^{(1)}=0
\end{array}\right\}, \\
L_{3 j}^{(0)}=\sqrt{\left(x_{3 j}^{(0)}\right)^{2}+\left(y_{3 j}^{(0)}\right)^{2}} .
\end{gathered}
$$

Positions of the mesh fabric and the SGS cables just after $1^{\text {st }}$ step of pulling are shown in Fig. 12.
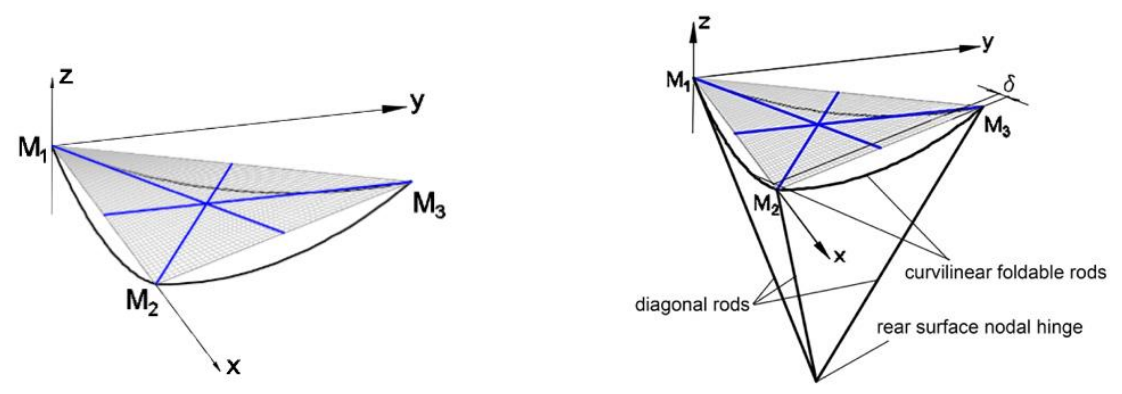

Fig. 12. Positions of the mesh fabric and the SGS cables just after $1^{\text {st }}$ step of pulling. 


\subsection{2 $2^{\text {nd }}$ step}

Displacements of the mesh nodes for the $2^{\text {nd }}$ step of deformation are shown in Fig. 13. In this case, nodes that belong to the flat triangle $M_{1} M_{2} M_{3}$ edges shift to the arcs corresponding to the curvilinear rods of the facet.

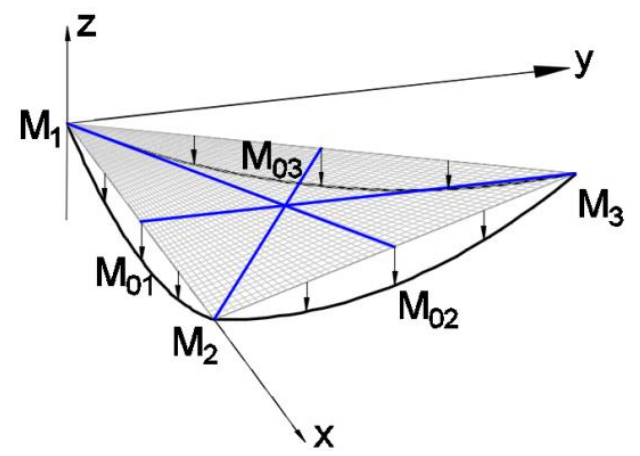

Fig. 13. $2^{\text {nd }}$ step of the knitted mesh fabric deformation.

Nodes $M_{i}$ that belong to line $M_{1} M_{2}$ shift along axis $M_{1} Z$ in compliance with the law of (6) (along the arrows in Fig. 13)

$$
\left.\begin{array}{c}
u_{1 i}^{(2)}=0 \\
v_{1 i}^{(2)}=0 \\
w_{1 i}^{(2)}=z_{1 c}-\sqrt{R_{1}^{2}+\left(x_{1 i}^{(1)}-x_{1 c}\right)^{2}}
\end{array}\right\},
$$

where $x_{1 c}, z_{1 c}$ are coordinates of the center of circle that includes arc $M_{1} M_{01} M_{2},\left(y_{1 c}=0\right)$, $R_{1}$ is the radius of the circle.

Nodes $M_{k}$ that belong to line $M_{2} M_{3}$ shift along axis $M_{1} Z$ in compliance with the law of (7) (along the arrows in Fig. 13)

$$
\left.\begin{array}{c}
u_{2 k}^{(2)}=0 \\
v_{2 k}^{(2)}=0 \\
w_{2 k}^{(2)}=z_{2 c}^{\prime \prime}-\sqrt{R_{2}^{2}-\left(x_{2 k}^{\prime \prime(1)}-x_{2 c}^{\prime \prime}\right)^{2}}
\end{array}\right\},
$$

where

$x_{2 k}^{\prime \prime(1)}=e_{11}^{\prime \prime}\left(x_{2 k}^{(1)}-x_{2}\right)+e_{12}^{\prime \prime}\left(y_{2 k}^{(1)}-y_{2}\right), x_{2 c}^{\prime \prime}=e_{11}^{\prime \prime}\left(x_{2 c}-x_{2}\right)+e_{12}^{\prime \prime}\left(y_{2 c}-y_{2}\right)$,

$z_{2 c}^{\prime \prime}=z_{2 c}, e_{11}^{\prime \prime}=\frac{x_{3}-x_{2}}{L_{2}^{(1)}}, e_{11}^{\prime \prime}=\frac{y_{3}-y_{2}}{L_{2}^{(1)}}$,

$x_{2}, y_{2}$ are coordinates of the point $M_{2}$,

$x_{3}, y_{3}$ are coordinates of the point $M_{3}$,

$x_{2 c}, y_{2 c}, z_{2 c}$ are coordinates of the center of circle that includes arc $M_{2} M_{02} M_{3}$,

$$
L_{2}^{(1)}=\sqrt{\left(x_{3}-x_{2}\right)^{2}+\left(y_{3}-y_{2}\right)^{2}} .
$$


Nodes $M_{j}$ that belong to line $M_{1} M_{3}$ shift along axis $M_{1} Z$ in compliance with the law of (8) (along the arrows in the Fig. 13)

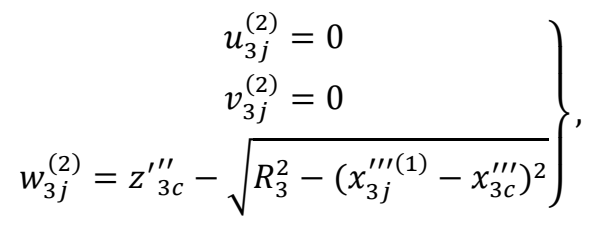

where

$x_{3 j}^{\prime \prime \prime}(1)=e_{11}^{\prime \prime \prime} x_{3 j}^{(1)}+e_{12}^{\prime \prime \prime} y_{3 j}^{(1)}, \quad x_{3 c}^{\prime \prime \prime}=e_{11}^{\prime \prime \prime} x_{3 c}+e_{12}^{\prime \prime \prime} y_{3 c}$,

$z_{2 c}^{\prime \prime \prime}=z_{3 c}, e_{11}^{\prime \prime \prime}=\frac{x_{3}}{L_{3}^{(1)}}, e_{11}^{\prime \prime}=\frac{y_{3}}{L_{3}^{(1)}}$,

$x_{3}, y_{3}$ are coordinates of the point $M_{3}$,

$x_{3 c}, y_{3 c}, z_{3 c}$ are coordinates of the center of circle that includes arc $M_{1} M_{03} M_{3}$,

$$
L_{3}^{(1)}=\sqrt{x_{3}^{2}+y_{3}^{2}}
$$

Positions of the mesh fabric and the SGS cables just after $2^{\text {nd }}$ step of pulling are shown in Fig. 14.
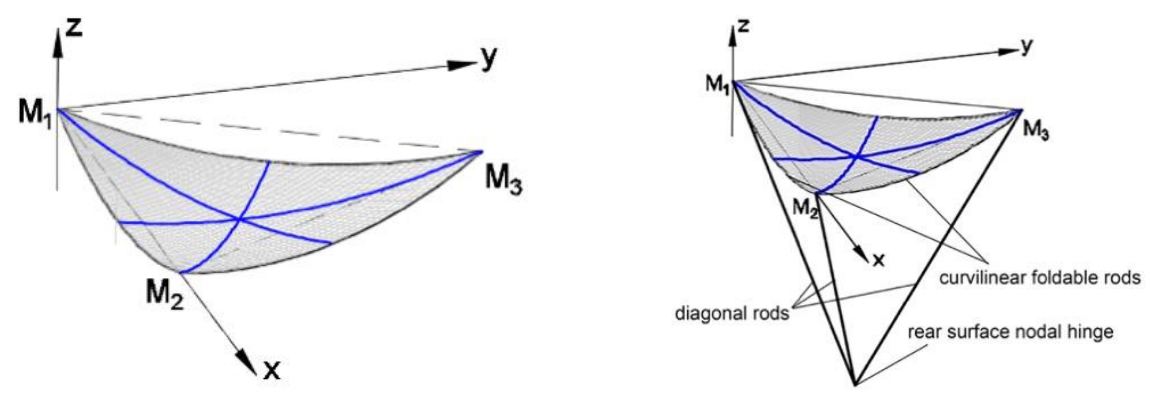

Fig. 14. Positions of the mesh fabric and the SGS cables just after $2^{\text {nd }}$ step of pulling.

\subsection{3 $3^{d}$ step}

Two variants of nodes shift of the SGS flexible cables mesh have been considered at $3 \mathrm{~d}$ step of the knitted fabric deformation (Fig. 15):

a) shift of the guy line connected to the node, where SGS cables intersect, is given (Fig. 15a)

b) additional set of six guy lines is introduced that are connected to the central guy line, which interconnects the node, where SGS cables intersect, with the hinge of the rear surface. Shift of the guy lines common node is given, which is equal to difference of $\mathrm{z}$ coordinate of this node and corresponding point, which belongs to the theoretical paraboloid. 


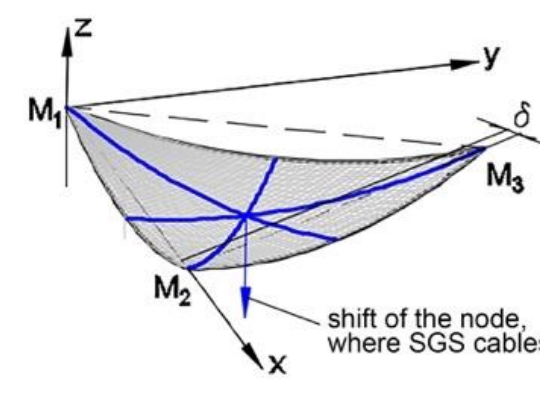

a)

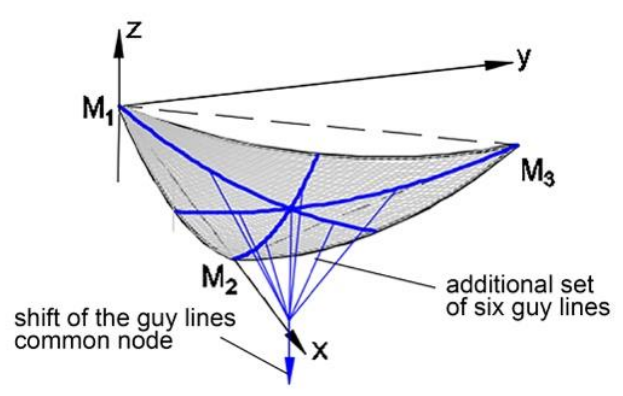

b)

Fig. 15. $3^{\mathrm{d}}$ step of the knitted mesh fabric deformation.

\subsection{Variants of performing of the root mean square deviation analysis and the stress analysis of the mesh fabric}

When performing the calculations, special attention is paid to defining the mesh fabric root mean square deviation (RMSD) from the theoretical paraboloid surface, which is based on customary in mathematical statistics RMSD concept. According this, RMSD value is defined as (9)

$$
R M S D=\sqrt{\frac{1}{S} \int_{S}[d(\boldsymbol{r})-\bar{d}]^{2} d S}, \quad \text { where } \bar{d}=\frac{1}{S} \int_{S} d(\boldsymbol{r}) d S
$$

After transformation, the formula for root mean square deviation $R M S D_{i}$ can be received, which is corresponding to each part of the reflecting surface $S_{i}(10)$

$$
R M S D_{i}=\sqrt{\overline{d_{l}^{2}}-\bar{d}_{\imath}^{2}}, \quad i=1,2,3, \cdots, N_{\Delta}
$$

where

$\overline{d_{l}^{2}}=\frac{1}{S_{i}} \int_{S_{i}} d_{i}^{2}(\boldsymbol{r}) d S_{i}, \quad \bar{d}=\frac{1}{S_{i}} \int_{S_{i}} d_{i}(\boldsymbol{r}) d S_{i}$,

$d_{i}(\boldsymbol{r})$ is the deviation of the point $M_{i}(\boldsymbol{r}) \in S_{i}$ from the theoretical paraboloid surface $S_{\text {par }}$, $\bar{d}_{i}$ is the mean value of deviation $d_{i}$ over the $S_{i}, \overline{d_{l}^{2}}$ is the mean value of squared deviation $d_{i}$ over the $S_{i}$.

The results of the single facet with various SGS flexible elements root mean square deviation analysis and stress analysis are given below.

While working on considered model of deformation of the mesh fabric with the SGS flexible elements, it is possible to vary the following parameters:

1) parameter $\delta$ defining initial sizes of the mesh fabric nesting, it is shown in Fig. 9. This parameter allows changing the meshed fabric stressed state;

2) arcs curvature, i.e. positions of the points defining the heights of arcs. The coordinates of the points can be found from the conditions of lying the points on the theoretical paraboloid surface or locating on outside of the paraboloid.

3) number and positions of the SGS cables. 


\subsubsection{Distribution of RMSD value along the module surface}

The estimation of RMSD value distribution along the module surface is shown in Fig. 16. Facets with the curvilinear foldable rods is considered being without SGS cables, under condition that the points corresponding to heights of arcs lie on the paraboloid surface.

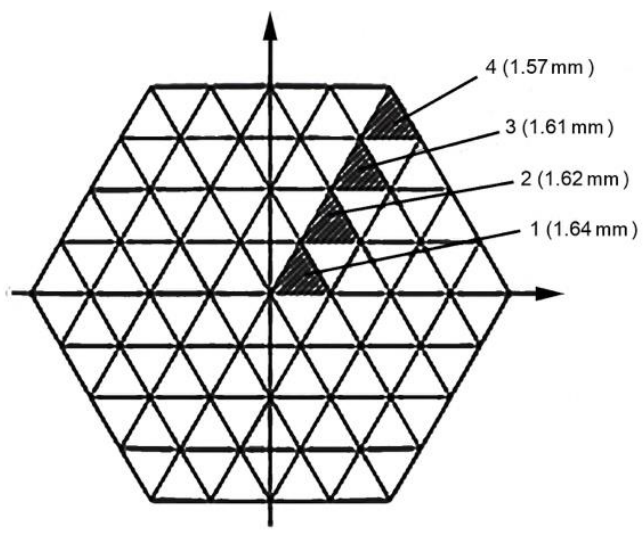

Fig. 16. Distribution of RMSD value along the module surface.

Following values for RMSD are found: $1.637 \mathrm{~mm}$ for facet $1,1.62 \mathrm{~mm}, 1.61 \mathrm{~mm}$, and $1.57 \mathrm{~mm}$ for facets 2,3 , and 4 respectively, which is explained by decreasing of the paraboloid surface curvature when moving away from its peak.

It should be noted, that RMSD of facet 1, on the assumption that foldable rods are rectilinear, is equal to $3.02 \mathrm{~mm}$, i.e. the facet is a flat triangle.

\subsubsection{Deformation of a single facet without SGS cables}

Here is calculation without the SGS cables, the foldable rods are curvilinear, points corresponding to the arcs heights are shifted by $2 \mathrm{~mm}$ towards the external normal to the paraboloid surface (Fig. 17). The RMSD value equal to $1.44 \mathrm{~mm}$ is obtained in this calculation.

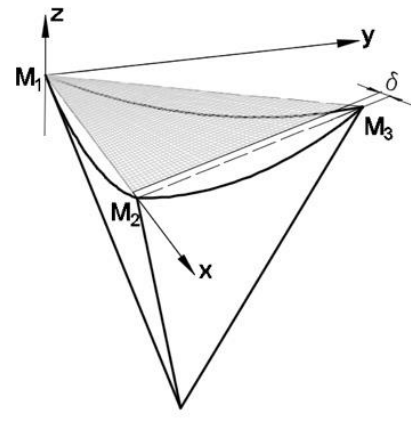

a)

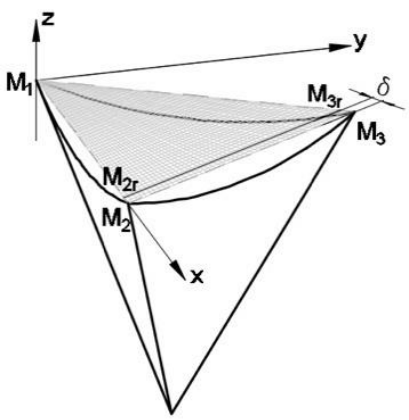

b)

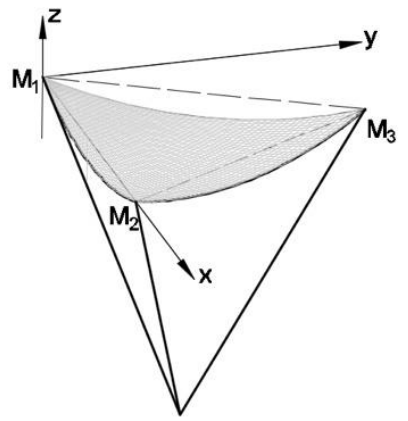

c)

Fig. 17. Points corresponding to the arcs heights are shifted by $2 \mathrm{~mm}$ towards the external normal to the paraboloid surface. 


\subsubsection{Deformation of a single facet enhanced by three SGS cables}

Here is calculation with the SGS cables, the foldable rods are curvilinear, points corresponding to the arcs heights lie on the paraboloid surface. Three flexible elements and a guy line are added (Fig. 18).

The RMSD values obtained in this calculation are next:

a) $R M S D=1.476 \mathrm{~mm}$. The point of the SGS cables common node doesn't lie on the paraboloid, and that corresponds to the guy line node shift by $(-11.7) \mathrm{mm}$.

b) $R M S D=0.985 \mathrm{~mm}$. The point of the SGS cables common node lies on the paraboloid, and that corresponds to the guy line node shift by $(-15.6) \mathrm{mm}$.

In the calculations, the SGS cables common node shift is accepted with minus sign, because of it occurs in the negative direction of axis $0 z$.

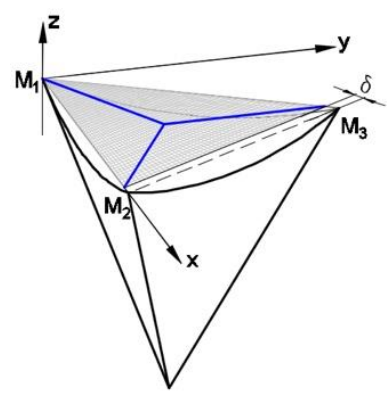

a)

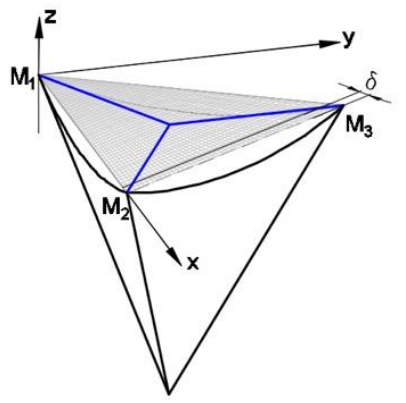

b)

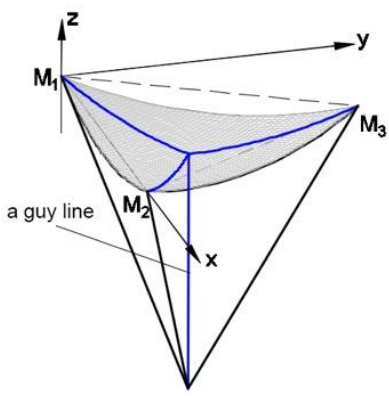

c)

Fig. 18. SGS consisting of three cables coming together at one node.

\subsubsection{Deformation of a single facet enhanced by three SGS cables and a set of guy lines}

Here is calculation with the SGS cables, the foldable rods are curvilinear, points corresponding to the arcs heights lie on the paraboloid surface.

Three flexible elements are carried out from vertices to opposite sides and a guy line are added (Fig. 19). The cables common node shift provides their placing on the paraboloid surface.

Following calculations of RMSD are carried out:

a) $R M S D=0.925 \mathrm{~mm}$. The point of the SGS cables common node lies on the paraboloid, and that corresponds to the guy line node shift by (-15.6) $\mathrm{mm}$ (Fig. 19c);

b) $R M S D=1.05 \mathrm{~mm}$. Additionally, points corresponding to the arcs heights are shifted by $2 \mathrm{~mm}$. This makes surface approximation to be worse (Fig. 19c)

c) Additional guy lines are entered, which have the common node connected with the central guy line (Fig. 19d):

$-R M S D=0.74 \mathrm{~mm}$, when $\delta=70 \mathrm{~mm}$ in the case of three additional guy lines;

$-R M S D=0.83 \mathrm{~mm}$, when $\delta=5 \mathrm{~mm}$ in the case of three additional guy lines;

$-R M S D=0.50 \mathrm{~mm}$, when $\delta=5 \mathrm{~mm}$ in the case of six additional guy lines; 


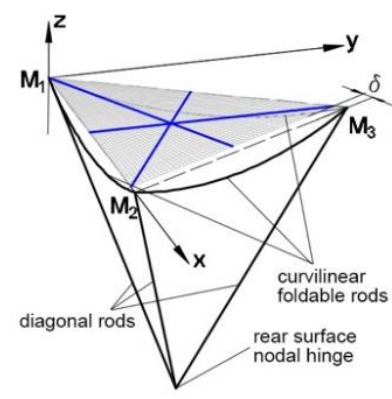

a)

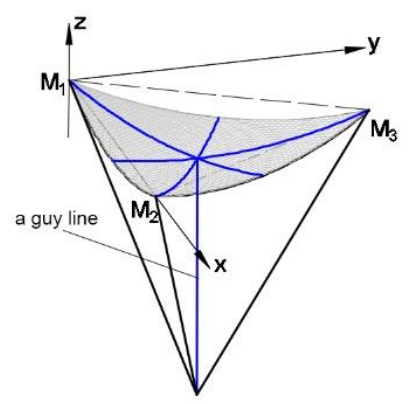

c)

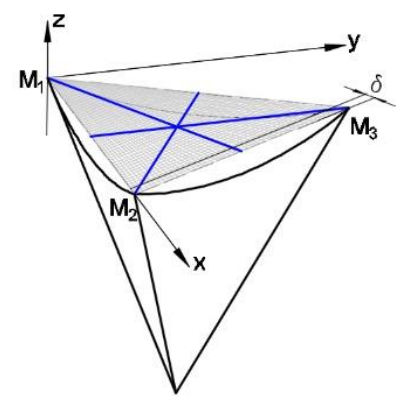

b)

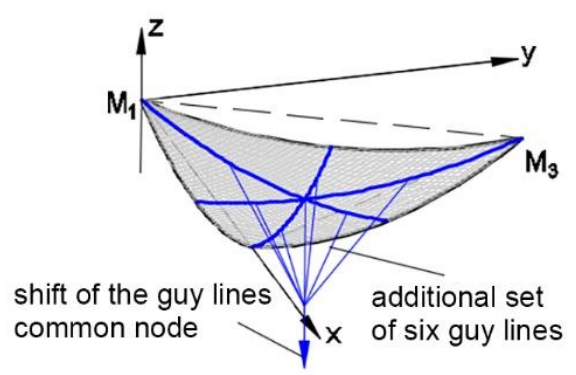

d)

Fig. 19. SGS consisting of three cables and additional guy lines.

\subsubsection{Deformation of a single facet with taking into account nonlinear properties of the SGS cables}

Here is the calculation variant when nonlinear way of the SGS cables deformation is taken into account. Deformation of the cables can be considered by two steps:

1) straightening of the cables, which is attended by friction forces action on mesh fabric, while it being deformed;

2) deformation of the cables.

To modeling gradual beginning of the SGS cables operation, nonlinear force characteristics of cables deformation is accepted, which is shown in Fig. 20.

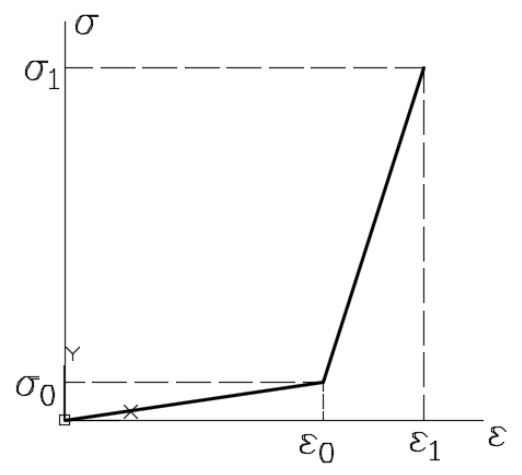

Fig. 20. Stress-strain curve $\sigma(\varepsilon)$ of the SGS cables. 
Following values of $\varepsilon_{0}, \varepsilon_{1}, \sigma_{0}, \sigma_{1}$ are accepted:

$\varepsilon_{0}=0,010 ; \sigma_{0}=1000 \mathrm{~Pa} ; \varepsilon_{1}=0,015 ; \sigma_{1}=3,6 \cdot 10^{7} \mathrm{~Pa} E=7,18 \cdot 10^{9} \mathrm{~Pa}$.

$\varepsilon_{1}$ value corresponds to $\delta=15 \mathrm{~mm}$, while $\varepsilon_{0}$ value corresponds to $\delta=10 \mathrm{~mm}$.

Deformation of the cables starts when current distance between free edge of the mesh fabric and line $M_{2} M_{3}$ at first deformation step makes up value by order of $5 \mathrm{~mm}$. Before this moment, the straightening of the cables appears, which is attended by friction forces action on mesh fabric, while it being deformed. This deformation step answers to section $\left[0, \varepsilon_{0}\right]$ at the stress-strain curve.

Described approach to the SGS cables deforming representation allows getting necessary mesh fabric tension and allowable stresses arising in the cables themselves.

The value of RMSD obtained in this calculation amounts $R M S D=0.27 \mathrm{~mm}$.

The greatest stresses arising in the cables amount $\sigma_{\max }=5.84 \mathrm{MPa}$, while allowable stress amounts $[\sigma]=(4.9 \div 6.8) \mathrm{MPa}$. Stresses arising in the mesh fabric amount $\sigma_{\text {max }}=0.086 \div 0.091 \mathrm{MPa}$, while operating stress amounts $\sigma_{o p} \approx 0.1 \mathrm{MPa}$.

Fig. 21a shows equivalent stresses $\sigma_{e}$ distribution along the knitted mesh fabric, while Figures $21 \mathrm{~b}$ and $21 \mathrm{c}$ show principal stresses $\sigma_{1}$ и $\sigma_{2}$ distribution.

The calculation results show that both of principal stresses arising in the mesh fabric are positive, therefore, it can be concluded that uniaxial areas in the mesh fabric aren't to be observed.

Fig. 21 shows stressed state of the knitted mesh fabric.

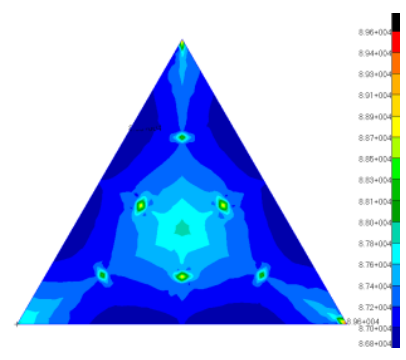

a)

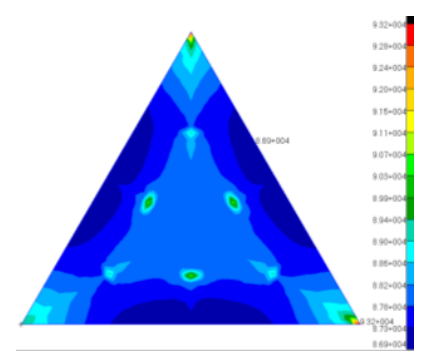

b)

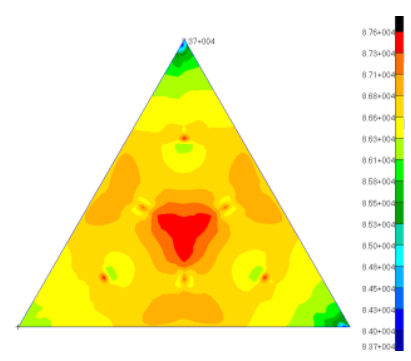

c)

Fig. 21. Stressed state of the knitted mesh fabric in $\mathrm{Pa}$ :

a) equivalent stresses $\sigma_{e}$ distribution,

b) principal stress $\sigma_{1}$ distribution, c) principal stress $\sigma_{2}$ distribution.

Distribution of magnitude of displacement vector $|\mathbf{u}|$ over the mesh fabric nodes and distribution of value $u_{z}$, which is projection of the displacement vector on the axis $0 z$, are shown in Fig. 22.

In the case in question, according the paraboloid surface curvature at the facet is small, deflections are determined as differences of z-coordinates of paraboloid and meshed surface, they are shown in Fig. 23 on the enlarged scale. At that, the plus sign corresponds to deflections along the inner normal of the paraboloid, while the minus sign corresponds to deflections along the outer one.

Positive values of the deflections surface answer to pillow effect when some areas of the surface are convex upwards. Light, convex downwards areas of the deflections surface answer to SGS cables effect, which in this case pull over the meshed surface towards the outer region of the paraboloid. The middle guy line provides its application point to be lying on the paraboloid surface. 


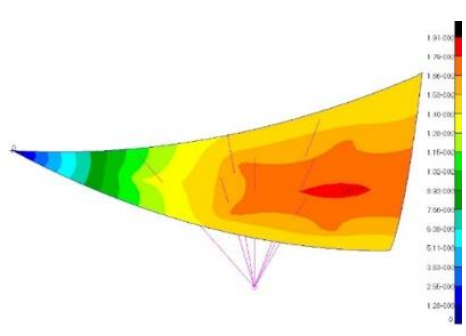

a)

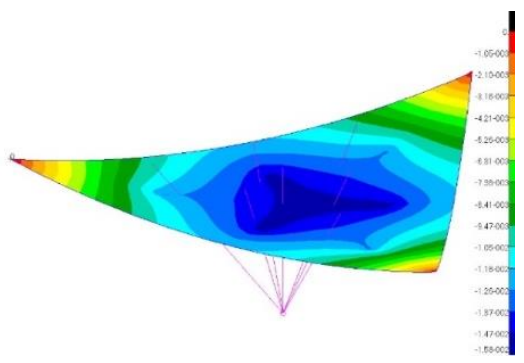

b)

Fig. 22. Distribution of knitted mesh fabric points displacements:

a) distribution of magnitude of displacement vector $|\mathbf{u}|$,

b) distribution of the displacement vector $|\mathbf{u}|$ projections $u_{z}$ on the axis $0 z$.

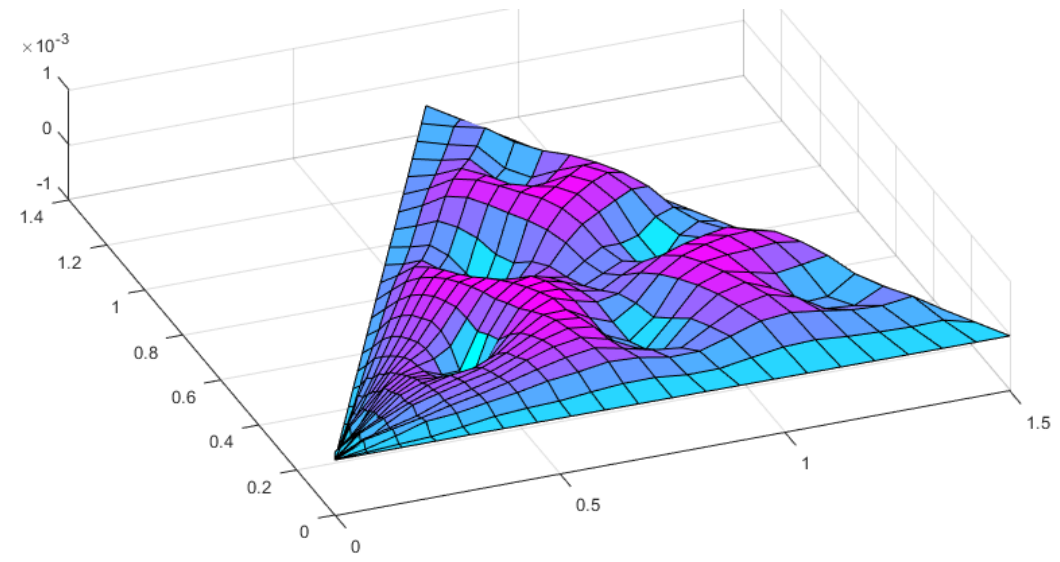

Fig. 23. Isometric view of the deflections surface.

The approach that is described above to perform the knitted mesh fabric stress analysis is used to calculate the surface shape of the real space calibration and adjustment sattelite (reflector), which have its reflecting surface geometry instrumentally measured.

\section{Calibration and adjustment satellite (reflector)}

For calibration and positioning purposes of ground radar staitions the calibration and adjustment satellites (CAS), which are launched to circumterrestrial orbits, are usable [21]. In the most simple case, to estimate the energy potential of a radar station, satellite holding only reflecting surface by given shape is usable. In particular, construction of a such satellite may represent the deployable spherical shell, reflecting surface of which is made from metallic knitted mesh fabric [22, 23] fixed to bearing deployable framework (Fig. 24).

Main CAS units are central telescopic bar containing the holding assemblies that serve to hold the bar in folded transport state, and the entity of pivotally interconnected rods in common with bar combining into the load-bearing foldable framework. The reflector framework is made of sets of rods, which are sequentially interconnected by flexible hinges and in unfolded state form into curvilinear spokes of the framework, which are aligned along the meridionals sections and the equatorial section of the spherical surface. Ends of 
meridional sets of rods (meridional ribs) are pivotally connected with the two pole hinges fixed to the ends of the telescopic bar.

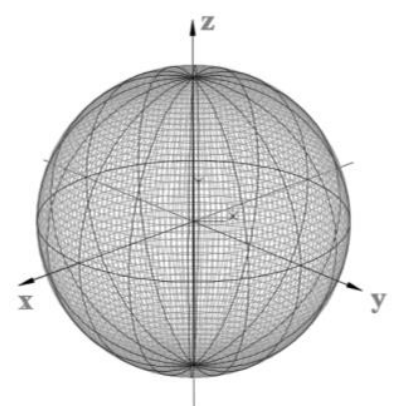

Fig. 24. General view of the reflector with fixed to it knitted mesh fabric.

Steps of CAS deployment and the CAS framework in the unfolded state are shown in Fig. 25.

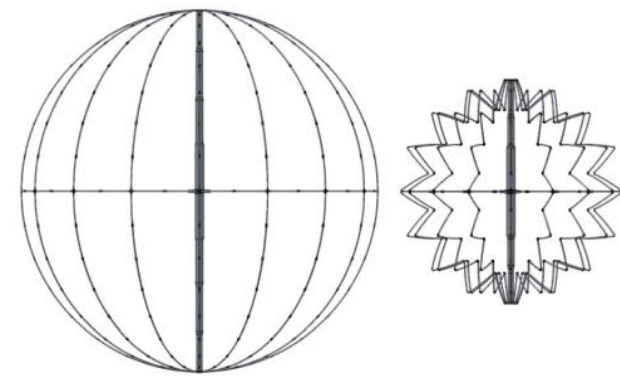

a)

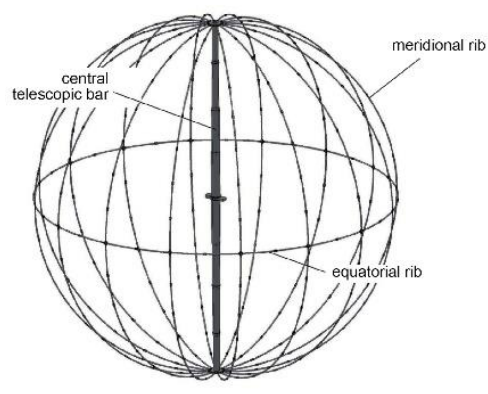

b)

Fig. 25. Steps of CAS deployment and the CAS framework in the unfolded state:

a) unfolded, intermediate and folded states of the reflector framework,

b) load-bearing framework in the unfolded state.

\subsection{Initial state}

Fig. 26 shows initial state of a reflector single lobe with radius $\mathrm{R}$, which is defined by angle $\alpha$. The angle $\alpha$ defines initial sizes of the lobe nesting, which is placed between two adjacent meridians of the reflector framework, i.e. there are known spherical and cartesian coordinates of the main points $M_{1}, M_{2}, M_{3}, M_{2 r}, M_{3 r}$.

Coordinates of points $M_{i}, M_{k}, M_{j}$ belonging to arcs $\bar{M}_{1} M_{2 r}, \bar{M}_{1} M_{3 r}$ and line $M_{2 r} M_{3 r}$ are defined by coordinates of the finite element mesh nodes.

1) Coordinates of points (11), which belong to the $\operatorname{arc} \bar{M}_{1} M_{2}(\varphi=0)$

$$
\left.\begin{array}{l}
M_{2}: x_{2}=R, y_{2}=0, z_{2}=0 \\
M_{2 r}: x_{2 r}=R \sin \theta_{2 r}, y_{2 r}=0, z_{2 r}=R \cos \theta_{2 r} \\
M_{i}: x_{i}=R \sin \theta_{i}, y_{i}=0, z_{i}=R \cos \theta_{i}
\end{array}\right\},
$$

where $\theta_{2 r}=\frac{\pi}{2}-\alpha$. 


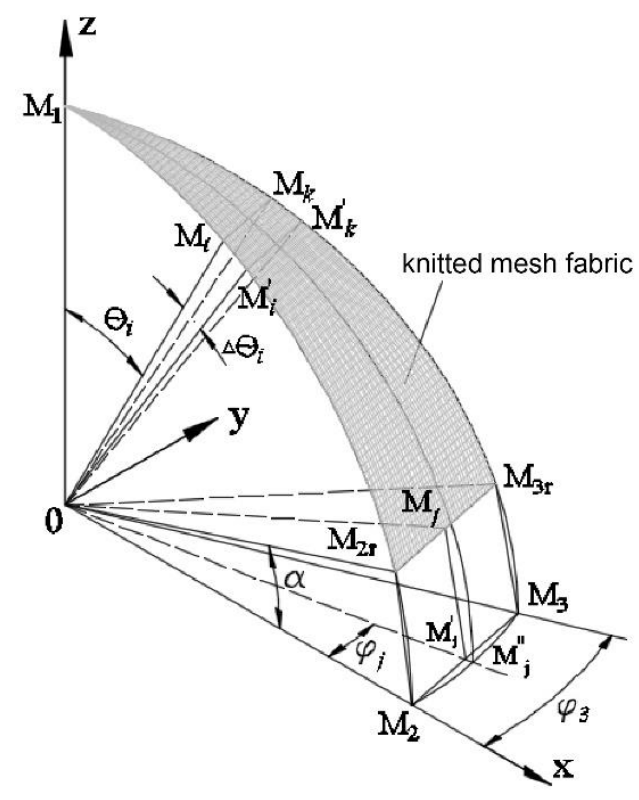

Fig. 26. Initial state of the mesh fabric of a single lobe.

2) Cartesian coordinates of the points $M_{j}(12)$

$$
\left.\begin{array}{l}
x_{j}=r_{j} \sin \theta_{j} \cos \varphi_{j} \\
y_{j}=r_{j} \sin \theta_{j} \sin \varphi_{j} \\
z_{j}=r_{j} \cos \varphi_{j}
\end{array}\right\} .
$$

The equation of the chord (line) $M_{2} M_{3}$ as a line being drawn through two given points (13)

$$
\left.\begin{array}{l}
M_{2}: x_{2}=R, y_{2}=0, z_{2}=0 \\
M_{3}: x_{3}=R \cos \varphi_{3}, y_{3}=R \sin \varphi_{3}, z_{i}=0
\end{array}\right\}
$$

is of the form (14)

$$
y=k_{3} x+b_{3}, \quad \text { where } k_{3}=\frac{y_{3}}{y_{3}-R}, b_{3}=-\frac{y_{3}}{x_{3}-R} .
$$

The equation of the line $0 M_{j}^{\prime}(15)$

$$
y=k_{j} x, \quad \text { where } k_{j}=\operatorname{tg} \varphi_{j} .
$$

By the system of linear equations (16)

$$
\left.\begin{array}{l}
y_{j}^{\prime}=k_{3} x_{j}^{\prime}+b_{3} \\
y_{j}^{\prime}=k_{j} x_{j}^{\prime}
\end{array}\right\},
$$

coordinates of the points $M_{j}^{\prime}\left(x_{j}^{\prime}, y_{j}^{\prime}, 0\right)(17)$, which belong to the chord $M_{2} M_{3}$, can be found

$$
x_{j}^{\prime}=\frac{b_{3}}{k_{j}-k_{3}}, y_{j}^{\prime}=\frac{k_{j} b_{3}}{k_{j}-k_{3}}, z_{j}^{\prime}=0 .
$$


Coordinates of the points $M_{j}^{\prime \prime}(18)$ lying on the equator

$$
x_{j}^{\prime \prime}=R \cos \varphi_{j}, y_{j}^{\prime \prime}=R \sin \varphi_{j}, z_{j}^{\prime \prime}=0
$$

3) Coordinates of the points, which belong to $\operatorname{arc} \bar{M}_{1} M_{3}\left(\varphi=\varphi_{3}\right)(19)$

$$
\left.\begin{array}{l}
M_{3}: x_{3}=R \cos \varphi_{3}, y_{3}=R \sin \varphi_{3}, z_{3}=0 \\
M_{3 r}: x_{3 r}=R \sin \theta_{3 r} \cos \varphi_{3}, y_{3 r}=R \sin \theta_{3 r} \sin \varphi_{3}, z_{3 r}=R \cos \theta_{3 r} \\
M_{k}: x_{k}=R \sin \theta_{k} \cos \varphi_{3}, y_{k}=R \sin \theta_{k} \sin \varphi_{3}, z_{k}=R \cos \theta_{k}
\end{array}\right\}
$$

where $\theta_{3 r}=\theta_{2 r}=\frac{\pi}{2}-\alpha$.

\subsection{Actual state}

Pulling of the lobe is made by one loading step.

This pulling variant is noticeable by the fact that nodes placed on the line $M_{2 r} M_{3 r}$ move directly to $\operatorname{arc} M_{2} M_{3}$ belonging to the equator.

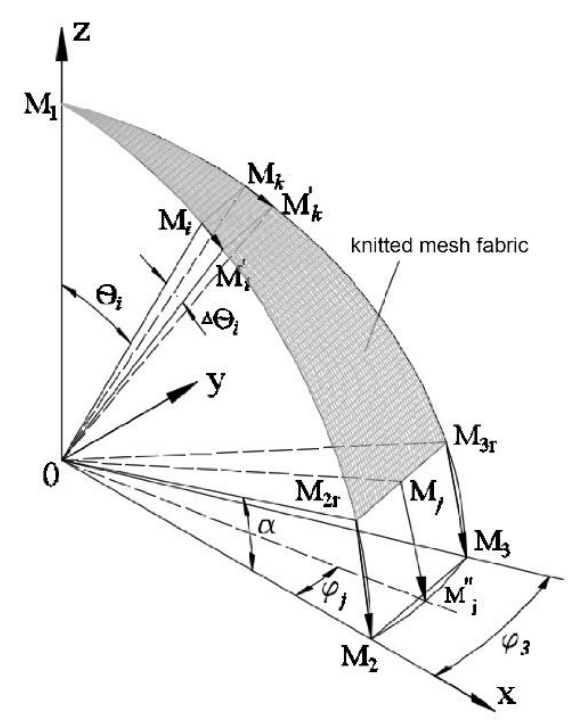

a)

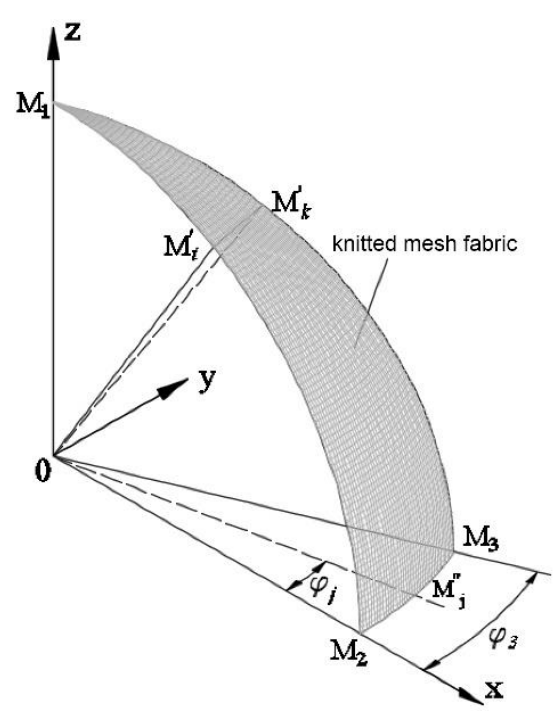

b)

Fig. 27. The mesh fabric pulling:

a) direction of mesh fabric main nodes moving,

b) mesh fabric actual state.

The displacement of the point $M_{2 r}$ in the Cartesian coordinate system is defined as (20)

$$
\mathbf{u}_{2 r}=\mathbf{r}_{2}-\mathbf{r}_{2 r}=\left(x_{2}-x_{2 r}\right) \mathbf{e}_{1}+\left(y_{2}-y_{2 r}\right) \mathbf{e}_{2}-\left(z_{2}-z_{2 r}\right) \mathbf{e}_{3}
$$

or $(21)$

$$
\left.\begin{array}{l}
u_{2 r}=R\left(1-\sin \theta_{2 r}\right) \\
v_{2 r}=0 \\
w_{2 r}=-R \cos \theta_{2 r}
\end{array}\right\}
$$


The displacement of the point $M_{3 r}$ in the Cartesian coordinate system is defined as (22)

$$
\mathbf{u}_{3 r}=\mathbf{r}_{3}-\mathbf{r}_{3 r}=\left(x_{3}-x_{3 r}\right) \mathbf{e}_{1}+\left(y_{3}-y_{3 r}\right) \mathbf{e}_{2}-\left(z_{3}-z_{3 r}\right) \mathbf{e}_{3}
$$

or (23)

$$
\left.\begin{array}{l}
u_{3 r}=R\left(1-\sin \theta_{3 r}\right) \cos \varphi_{3 r} \\
v_{3 r}=R\left(1-\sin \theta_{3 r}\right) \sin \varphi_{3 r} \\
w_{3 r}=-R \cos \theta_{3 r}
\end{array}\right\}
$$

Points $M_{i}$ shift along the $\operatorname{arc} \bar{M}_{1} M_{2}$ to positions $M_{i}^{\prime}$. Then, in the Cartesian coordinate system (24)

$$
\mathbf{u}_{i}=\mathbf{r}_{i}^{\prime}-\mathbf{r}_{i}=\left(x_{i}^{\prime}-x_{i}\right) \mathbf{e}_{1}+\left(y_{i}^{\prime}-y_{i}\right) \mathbf{e}_{2}-\left(z_{i}^{\prime}-z_{i}\right) \mathbf{e}_{3}
$$

or (25)

$$
\left.\begin{array}{l}
u_{i}=R\left[\sin \left(\theta_{i}+\Delta \theta_{i}\right)-\sin \theta_{i}\right] \\
v_{i}=0 \\
w_{i}=R\left[\cos \left(\theta_{i}+\Delta \theta_{i}\right)-\cos \theta_{i}\right]
\end{array}\right\} .
$$

On proportionality of nodes displacement along the meridian

$$
\frac{\Delta \theta_{i}}{\alpha}=\frac{l_{i}}{l_{2 r}}=\frac{R \theta_{i}}{R \theta_{2 r}}=\frac{\theta_{i}}{\theta_{2 r}},
$$

angular displacement $\Delta \theta_{i}$ of the point $M_{i}$ can be found

$$
\Delta \theta_{i}=\frac{\theta_{i}}{\theta_{2 r}} \alpha .
$$

The same way, the displacements of the points $M_{k}$ in the Cartesian coordinate system can be found (27)

$$
\mathbf{u}_{k}=\mathbf{r}_{k}^{\prime}-\mathbf{r}_{k}=\left(x_{k}^{\prime}-x_{k}\right) \mathbf{e}_{1}+\left(y_{k}^{\prime}-y_{k}\right) \mathbf{e}_{2}-\left(z_{k}^{\prime}-z_{k}\right) \mathbf{e}_{3}
$$

or in the coordinate representation (28)

$$
\left.\begin{array}{l}
u_{k}=R\left[\sin \left(\theta_{k}+\Delta \theta_{k}\right)-\sin \theta_{k}\right] \cos \varphi_{k} \\
v_{k}=R\left[\sin \left(\theta_{k}+\Delta \theta_{k}\right)-\sin \theta_{k}\right] \sin \varphi_{k} \\
w_{k}=R\left[\cos \left(\theta_{k}+\Delta \theta_{k}\right)-\cos \theta_{k}\right]
\end{array}\right\},
$$

where

$$
\Delta \theta_{k}=\frac{\theta_{k}}{\theta_{3 r}} \alpha
$$

The displacements of the points $M_{j}(29)$

$$
\mathbf{u}_{j}=\mathbf{r}_{j}^{\prime \prime}-\mathbf{r}_{j}=\left(x_{j}^{\prime \prime}-x_{j}\right) \mathbf{e}_{1}+\left(y_{j}^{\prime \prime}-y_{j}\right) \mathbf{e}_{2}+\left(z_{j}^{\prime \prime}-z_{j}\right) \mathbf{e}_{3} .
$$

Since

$$
x_{j}^{\prime \prime}=R \cos \varphi_{j}, y_{j}^{\prime \prime}=R \sin \varphi_{j}, z_{j}^{\prime \prime}=0,
$$




$$
\left.\begin{array}{l}
x_{j}=r_{j} \sin \theta_{j} \cos \varphi_{j} \\
y_{j}=r_{j} \sin \theta_{j} \sin \varphi_{j} \\
z_{j}=r_{j} \cos \varphi_{j}
\end{array}\right\},
$$

then components of the displacements of points $M_{j}$ in the spherical coordinate system become (30)

$$
\left.\begin{array}{l}
u_{j}=\left(R-r_{j} \sin \theta_{j}\right) \cos \varphi_{j} \\
v_{j}=\left(R-r_{j} \sin \theta_{j}\right) \sin \varphi_{j} \\
w_{j}=-r_{j} \cos \varphi_{j}
\end{array}\right\} .
$$

\subsection{Calculation results}

Calculations are carried out for a single reflector lobe with the radius of $1.08 \mathrm{~m}$ and the mouth angle equal to 22.5. The knitted mesh fabric of the reflector with thikness of $150 \mu \mathrm{m}$ is made of material Atlas-Atlas with the mechanical properties $E=15 \cdot 10^{4} \mathrm{~Pa}, v=0.4$.

Pulling of the lobe is modeled by stretching along the meridians by one loading step.

Calculations are carried out for different length of the lobe nesting in the meridional direction. The length of the lobe nesting is specified by the value of angle $\alpha$ (Fig. 26).

Finite element model of the lobe is mesh of 390 finite elements, 380 of which are Quad4 type and 10 elements are Tria3 type.

Fig. 28 shows deformed state of a single reflector lobe, when $\alpha=10^{\circ}$. Initial nesting of the lobe is shown in Fig. 28a in the form of finite element mesh. Fig. 28b shows deformed state of a single lobe, which has dual curvature, on the enlarged scale.

Fig. 29a shows lobe stressed state and the scale of equivalent stresses.

Calculations show that maximum deflection $\delta_{\max }$ of calculated shape of the mesh fabric from theoretical sphere for the different values of angle $\alpha$ on average makes up value by order of $37.3 \mathrm{~mm}$, which differs from experimentally measured deflection of shape of the real reflector by $14 \%$. It should be noted, that maximum values of deflection in the calculations and in the experiment practically answer to the same values of angles $\theta$ and $\varphi$.

Table 2. Maximum deflections of the mesh fabric under the different characteristics of pulling.

\begin{tabular}{|c|c|c|c|c|}
\hline$\alpha, \mathrm{deg}$ & $\varphi, \mathrm{deg}$ & $\theta, \mathrm{deg}$ & $\delta_{\max }, \mathrm{mm}$ & $\%$ \\
\hline 7.25 & 11.3 & 72.0 & 36.8 & 15.0 \\
\hline 9.0 & 11.3 & 71.5 & 37.2 & 14.5 \\
\hline 10.0 & 11.3 & 71.5 & 37.3 & 14.3 \\
\hline 11.0 & 11.3 & 71.5 & 37.5 & 13.8 \\
\hline 15.0 & 11.3 & 72.0 & 37.7 & 13.3 \\
\hline Experimental data & 11.3 & 75.0 & 43.5 & \\
\hline
\end{tabular}

Table 3. Stressed state of the mesh fabric under the different characteristics of pulling.

\begin{tabular}{|c|c|c|c|c|}
\hline$\alpha, \mathrm{deg}$ & $\sigma_{x} \cdot 10^{-4}, \mathrm{~Pa}$ & $\sigma_{y} \cdot 10^{-4}, \mathrm{~Pa}$ & $T_{x}, \mathrm{gf} / \mathrm{cm}$ & $T_{y}, \mathrm{gf} / \mathrm{cm}$ \\
\hline 7.25 & $0.82-2.19$ & $1.15-2.19$ & $1.23-3.29$ & $1.73-3.29$ \\
\hline 9.0 & $1.22-2.78$ & $1.40-2.78$ & $1.83-4.17$ & $2.10-4.17$ \\
\hline 9.9 & $1.45-3.09$ & $1.53-3.09$ & $2.18-4.63$ & $2.30-4.63$ \\
\hline 10.0 & $-0.92-19.3$ & $-0.74-20.4$ & $0.00-29.0$ & $0.00-30.6$ \\
\hline 10.1 & $1.50-3.16$ & $1.56-3.16$ & $2.25-4.74$ & $2.34-4.74$ \\
\hline 11.0 & $1.74-3.48$ & $1.70-3.48$ & $2.61-5.22$ & $2.55-5.22$ \\
\hline 15.0 & $2.95-5.00$ & $2.37-5.00$ & $4.43-7.50$ & $3.56-7.50$ \\
\hline
\end{tabular}


As regards the stresses (Fig. 29a), it is evident from the Table 3 that when varying of angle $\alpha$ value, it can be provided the pulling of the mesh fabric to allowable amounts of membrane forces $T_{x}$ и $T_{y}$.

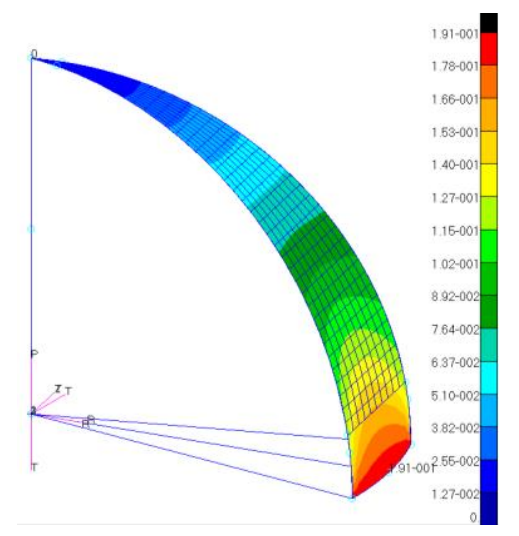

a)

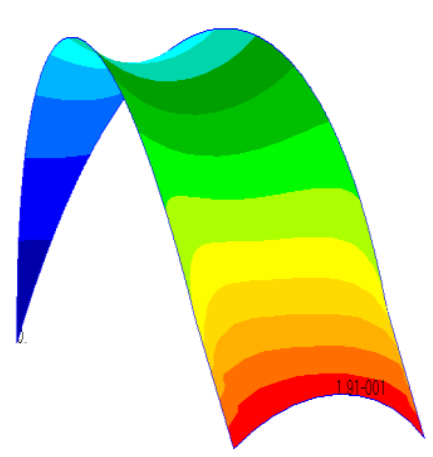

b)

Fig. 28. Deformed state of a single lobe:

a) deformed state with indication of initial state in the form of finite element mesh, b) deformed lobe on the enlarged scale.

Fig. 29b shows the reflecting surface shape of the CAS with radius of $1.08 \mathrm{~m}$ plotted by experimental data.

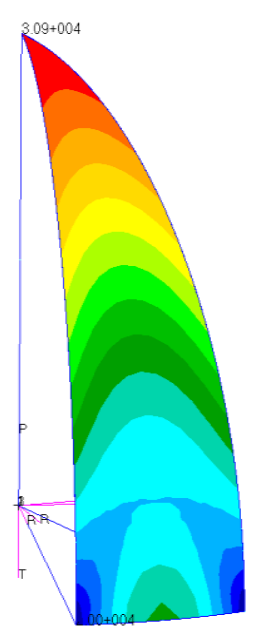

a)

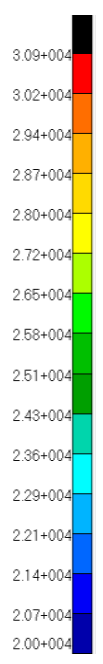

)

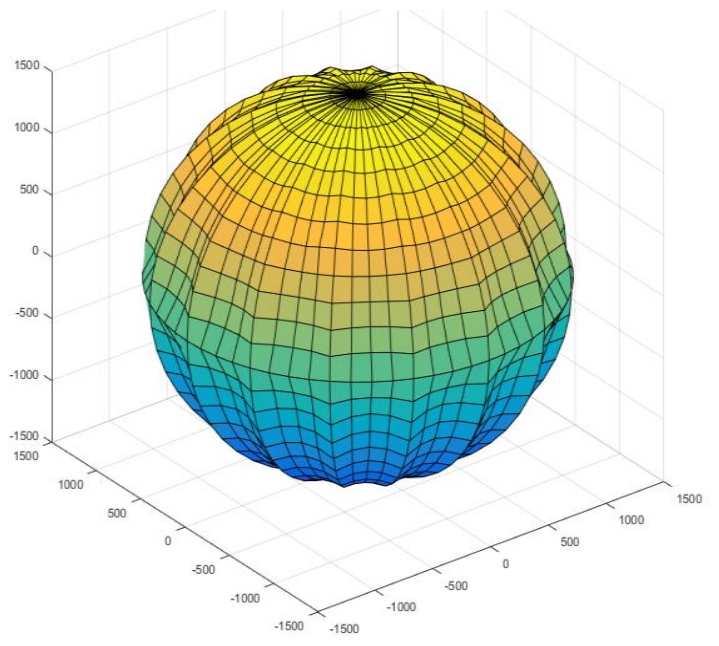

b)

Fig. 29. Stressed state of a single lobe:

a) stressed state of a single lobe, $\sigma_{e}, \mathrm{~Pa}$,

б) quasispherical shape of the reflector with diameter of $2 \mathrm{~m}$ plotted by experimental data.

Fig. 30a and 30b show distribution of principal stresses $\sigma_{1}$ и $\sigma_{2}$ over a single lobe of shell reflector with quasispherical shape after the knitted mesh fabric being pulled on the rigid frame. The calculation results show that both of principal stresses arising in the lobe 
are positive, therefore, it can be concluded, that uniaxial areas in the mesh fabric aren't to be observed. At that, it should be noted, that circumferential principal stress $\sigma_{2}$ takes minimum value in the equatorial region, where a meridian meets the equator. It is the region, where the mesh fabric pleats can appear when parameters of the mesh fabric pulling on the frame are different from the considered in this work.

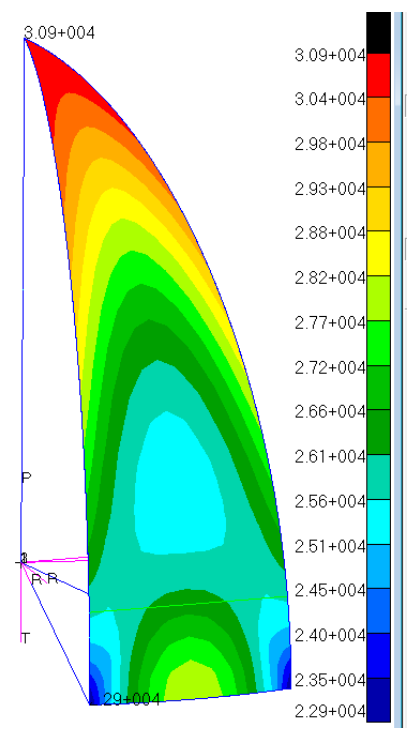

a)

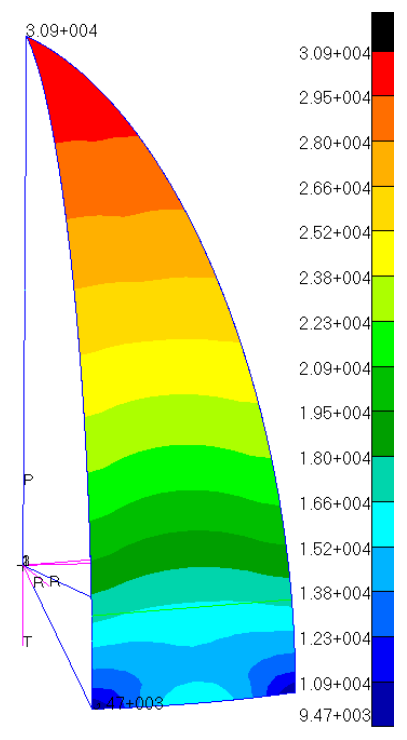

b)

Fig. 30. Principal stresses distribution:

over the reflector shell with quasispherical shape lobe,

a) principal stress $\left.\sigma_{1}, \mathrm{~Pa}, \mathrm{~b}\right)$ principal stress $\sigma_{2}, \mathrm{~Pa}$.

\section{Conclusion}

The numerical algorithm of calculation of stressed and deformed state of the reflecting mesh fabric step-by-step being pulled on the bearing frame, based on finite elements method, is proposed in this work.

It is shown that sufficient approximation precision of facets mesh reflecting surface is achieved by using flexible cables to the surface shaping.

Analyzing when having obtained results, it is possible to conclude what grade obtained shapes of reflecting surfaces are precise with, and to formulate variants of constructions, which would allow to obtain the facet surface shape with required accuracy, and in the wake, the whole reflector surface with the required precision.

Numerical investigation of the reflecting surface calculations of the full-scaled model of spherical calibration and adjustment satellite shows satisfactory qualitative and quantitative matching of calculation results with the experimental data.

Presented in this work investigation results allow to conclude that proposed algorithm can be practically usable to calculate stressed and deformed state of the reflecting surface made of knitted mesh fabric being pulled on the curvilinear frame when design calculations are carried out. 


\section{References}

1. G. Tibert, Deployable tensegrity structures for space applications, Doct. Thes. (2002)

2. A.I. Velichko, S.V. Belov, S.V. Ponomarev, Reshetnev readings, 1, 61-62 (2013)

3. S.V. Ponomarev, Tomsk St. Univ. J. of Mat. and Mech., 4, 110-119 (2011)

4. J. Santiago-Prowald, CalTech-KISS Large Space Apertures Workshop (2008)

5. J. Santiago-Prowald, M. Such-Taboada, http://www.upv.es/upl/U0532291.pdf (2010)

6. M. Thomson, AIAA 2002-2032 (2002)

7. S. Pivnenko, O.S. Kim, J. M. Nielsen, O. Breinbjerg, K. Pontoppidan, A. Østergaard, C.C. Lin, CEAS Sp. J., 5, 3-4, 211-219 (2013)

8. I. Khalimanovich, V.V. Shal'kov, S.V. Ponomarev, V.G. Butov, M.S. Buhtyak, V.A. Solonenko, A.A. Yashuk, Reshetnev readings, 1, 88-89 (2009)

9. V.V. Vozov, E.K. Cherkashina, V.V. Shalkov, D.O. Shendalev, Reshetnev readings, 1, 50-51 (2011)

10. J. Fager, R. Garriott, IEEE Trans. on Ant. and Prop. J., 17, 4, 452-458 (1969)

11. R.E. Freeland, R.G. Helms, CalTech-KISS Large Space Apertures Workshop (2008)

12. Kees van 't Klooster, L. Gurvits, Tijdschrift van het NERG, 67, 4 135-144 (2002)

13. S.E. Zaytsev, Kosmicheskiy apparat-nositel' RSA "Kondor-E" kak osnova radiolokatsyonnoy kosmicheskoy sistemy "Kondor-FKA" [Spacecraft Kondor-E as a basis of the radar space system "Kondor-FCA"], VI Vserossiyskiye Armandovskiye chteniya, 424-431 (2016)

14. Satoru Ozawa, CalTech-KISS Large Space Apertures Workshop (2008)

15. Y. Murata, The 9th European VLBI Network Symposium on The role of VLBI in the Golden Age for Radio Astronomy and EVN Users Meeting, 72 (2009)

16. K. Higuchi, N. Kishimoto, A. Meguro, H. Tanaka, M. Yoshihara, and Sh. Iikura, 50th AIAA/ASME/ASCE/AHS/ASC Structures, Structural Dynamics, and Materials Conference, AIAA 2009-2609 (2009)

17. Y. Murata, H. Hirabayashi, M.C. Natori, T.Umemoto, K.Asada, S.Iikura, and The next generation space VLBI working group, Proceedings of the XXVIIIth URSI General Assembly in New Delhi (2005)

18. K. Shintate, K. Terada, M. Usui, A. Tsujihata, and A. Miyasaka, J. Nat. Inst. Inf. and Comm. Techn., 49, 27-32 (2003)

19. N.M. Feizulla, Yu.A. Kisanov. Osobennosti formoobrazovaniya poverkhnosti kosmicheskikh antenn s setchatym otrazhatelem [Features of surface shaping of space antennas with meshed reflector], Antenny, Publ. Radio i Sviaz', 107-115 (1987)

20. W.B. Fichter, AIAA J., 22, 11 1679-1684 (1984)

21. G.I. Goziumov, Issues of radio electronics. Radar equipment series, 4, 53-67 (2002)

22. Yu.A. Kisanov, N.M. Feizulla, L.A. Kudriavin, V.A. Zavaruev, Antennas: collection of scientific papers, 29, edited by A.A. Pistolkors, Publ. Radio i Sviaz', 20-25, (1981)

23. Yu.N. Bugaev and others, Patent RU(11) 2396 649(13) C1 (2009) 